九州大学学術情報リポジトリ

Kyushu University Institutional Repository

\title{
Threshold of Enzymatic Reaction System, Further Studies with a Simplified Model
}

Okuyama, Kaoru

Laboratory of Sericultural Chemistry, Faculty of Agriculture, Kyushu University

Okamoto, Masahiro

Laboratory of Sericultural Chemistry, Faculty of Agriculture, Kyushu University

Aso, Yoichi

Laboratory of Sericultural Chemistry, Faculty of Agriculture, Kyushu University

Hayashi, Katsuya

Laboratory of Sericultural Chemistry, Faculty of Agriculture, Kyushu University

https://doi.org/10.5109/23661

出版情報：九州大学大学院農学研究院紀要. 22 (3)，pp. 153-160，1978-07. Kyushu University バージョン：

権利関係 : 


\title{
Threshold of Enzymatic Reaction System, Further Studies with a Simplified Model
}

\author{
Kaoru Okuyama, Masahiro Okamoto, Yoichi Aso and Katsuya Hayashi \\ Laboratory of Sericultural Chemistry, Faculty of Agriculture, \\ Kyushu University 46-02, Fukuoka 812
}

(Received November 24, 1977)

\begin{abstract}
By means of digital simulation, the saturation of threshold-type responses has been observed with a model system composed of several emzymatic reactions (Okuyama et al.. 1977). The model included rate constants which depended upon the product concentration:

In the present study, this dependency was eliminated in order to simplify the model, but the saturation was attained again. It was found that a feed-forward circuit, which was still set in the simplifed model, played an important role in establishing the saturated state. In order to search the molecular mechanism of the feed-forward loop, it was assumed that the loop was composed of a subsystem equilibrating between active and inactive forms of an enzyme. Contrary to expectation, thesaturation of threshold-type response was achieved only when the equilibrium of the above enzymatic system favored greatly thr inactive form.
\end{abstract}

\section{INTRODUCTION}

Threshold behavior in response to input information has been found in many biological processes and tried to clarify in many different ways. For example, Nimmo and Bauermeister (1976) recently proposed a cyclic model for drug-receptor interaction, in which receptors were thought of as being present in either of two states, an active conformation $(R)$ or an inactive one $(T)$, and showed a sigmoidal relationship between the concentration of the drug $(X)$ and the maximum response $\left(R X_{\text {matx }}\right)$ under limited conditions. Lewis et al. (1977) studied many pattern-forming processes in development in relation to the threshold concentration of positional information, and reported that a simple kinetic model based upon positive feedback could account both for memory and for discontinuity in the pattern of cell states. For an enzymatic reaction an allosteric mechanism (Monod et al., 1965) and an analogous model (Walter et al., 1967) have been proposed in order to explain the sigmoidal relationships between the concentration of substrate and the fractional saturation of enzyme with the substrate.

In the previous paper from our laboratory (Okuyama et al., 1976), it was reported that a threshold-type response was obtained by digital simulation of an enzymatic reaction system which was derived from a model previously proposed by Ličko (1972). Moreover, the saturated state could be attained in the threshold-type response by the model modified to contain two feedback loops and 
a feed-forward circuit (Okuyama et al., 1977). The effects of the feed-forward path on the saturation of the response were larger than those of the feedback loops because of the saturation of product concentration with input. Therefore, in the present study the model was simplified by eliminating the feedback paths. By this model the saturated state of threshold-type response could also be attained. Furthermore, it was tried to replace feed-forward path by a simple enzymatic equilibrium subsystem, and the latter subsystem was characterized by simulation in order to obtain an insight into the molecular mechanism of the feed-forward reaction.

\section{COMPUTATION}

Simulation by means of a digital computer was performed with Models $\mathrm{S}$ and T shown in Fig. 1. Model A in Fig. 1 was the same as step IV in the previous paper (Okuyama et al., 1977), and showed a sigmoidal relationship between an initial concentration of $\mathrm{X}\left(X_{0}\right)$ and a steady-state value of $Y\left(Y_{\infty}\right)$. Model $S$ is designed as a simplified form of Model $A$, in which the feed back loops were eliminated, and Model $T$ contains a subscheme replacing the positive feed-forward in Model S. It was assumed that the enzyme in the subscheme has both an inert form $(\boldsymbol{E}$,$) and an active form \left(E_{i}\right)$. The inert enzyme is converted into the active form by stimulation due to $X$. The active enzyme promotes the consumption of $Y$ and then transforms spontaneously into the inert form.

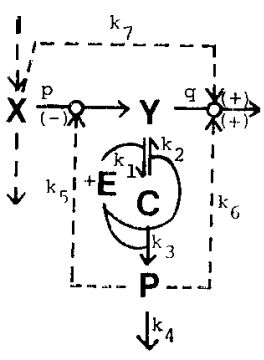

MODE. $\Lambda$

(1)

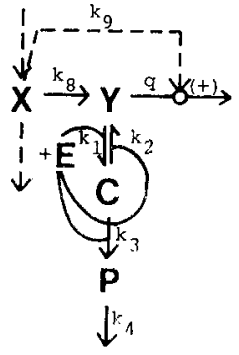

MODEL S

(2)

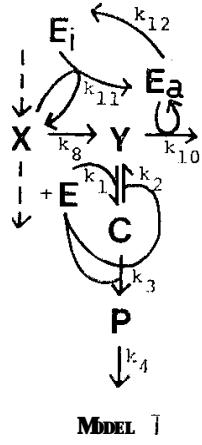

(3)

Fig. 1. Model systems used in theprevious study (1) and used in the present study (2), (3).

The rate equations for Model $A$ can be written as follows :

$$
\begin{aligned}
& \frac{d Y(t)}{d t}=\left\{p-k_{3} P(t)\right\} X_{0}-k_{1} E(t) Y(t)+k_{2} C(t)-q\left\{k_{6} P(t) k_{7}\right\} X_{0} Y(t) \\
& \frac{d C(t)}{d t}=k_{1} E(t) Y(t)-k_{2} C(t)-k_{3} C(t)
\end{aligned}
$$




$$
\begin{aligned}
& d E(t)=-k_{1} E(t) Y(t)+k_{2} C(t)+k_{3} C(t) \\
& d t \\
& \frac{d P(t)}{d t}=k_{3} C(t)-k_{1} P(t)
\end{aligned}
$$

where $X(t)$ was assumed to be kept at its initial concentration as has been shown in the previous paper (Okuyama et al., 1976). The initial concentrations were assumed to be $\mathrm{Y}(0)=\mathrm{C}(0)=P(0)=0$ and $\mathrm{E}(0)=0.2$ in arbitrary units. The values of rate constants were chosen as follows : $p=1.0, q=0.05$, $k_{1}=400.0, k_{2}=35.0, k_{3}=5.0, k_{4}=2.0, k_{5}=0.1, k_{6}=1.0, k_{7}=1.0 \quad$ (arbitrary units). These values were the same as those used in computation of Step IV and gave a saturated threshold (curve B) in the previous paper.

For Model S, the rate equation of the $\mathrm{Y}$ concentration is written as,

$$
\begin{gathered}
d Y(t) \\
d t
\end{gathered}=k_{8} X_{0}-k_{1} E(t) Y(t)+k_{3} C(t)-q k_{3} X_{0} Y(t)
$$

and the other rate equations are the same as those for Model A.

$$
\left.\begin{array}{l}
\mathrm{k},=p-k_{5} P(t) \\
k_{9}=k_{6} P(t) k_{7}
\end{array}\right\}
$$

If equation (3) is established, Models $\mathrm{A}$ and $\mathrm{S}$ may have the same response to input information. Substituting the $P_{\infty}$ value (which was the calculated steady-state value of product $P$ for Model A) for $P(t)$ in equation (3) yields the $k_{\text {si }}$ and $k_{9 \omega}$ values for each input. From the results obtained, approximated values of $k_{s}=1.0$ and $k_{9}=0$. 5 were adapted for numerical calculation of the rate equations for Model $\mathrm{S}$. The threshold $\theta$ did not change because $p=k_{k}=$ 1. 0 and $\theta=k_{3} E_{0} / p=k_{3} E_{0} / k_{8}=1$. 0 .

The rate equations on $\mathrm{Y}, E_{u}$ and $E_{i}$ for Model $\mathrm{T}$ may be written as

$$
\begin{aligned}
& \frac{d Y(t)}{d t}=k_{8} X_{0}-k_{1} E(t) Y(t)+k_{2} C(t)-k_{10} E_{a}(t) Y(t) \\
& \frac{d E_{i}(t)}{d t}=-k_{11} E_{i}(t) X_{0}+k_{12} E_{u}(t) \\
& \frac{d E_{a}(t)}{d t}=k_{11} E_{i}(t) X_{0}-k_{12} E_{a}(t)
\end{aligned}
$$

The other rate equations were the same as those for Model A. The additional initial concentrations were assumed to be $E,(O)=O .2$ and $E,(O)=0$ in arbitrary units. From equations (2) and (4), it is expected that Model $\mathrm{T}$ would behave in the same manner as Model $\mathrm{S}$, if it is assumed that the following relationship holds always true.

$$
k_{10} E_{a}(t)=q k_{9} X_{0}
$$

In Model $\mathrm{T}$, simulations were performed with changing the value of equilibrium constant $K$ 


$$
K=\frac{\left[E_{a \infty}\right]}{\left[X_{10}\right]\left[E_{i \infty}\right]}=\frac{k_{11}}{k_{12}}
$$

at $k_{8}=1.0$ as above, in order to obtain a relationship among the values of $K$, $E_{a \infty}, Y_{\infty}$, and $X_{0}$. From the results, the value of $k_{10}$ was obtained from equation(5) using the value of $E_{a \infty}$ at $X_{0}=10$ for each value of $K$ and $k_{9}=0$. 5. Then computations were performed for each set of $K$ and $k_{10}$ and the relationships between $X_{0}$ and $Y_{\infty}$ in Model $\mathrm{T}$ were compared with those between $X_{0}$ and $Y_{\infty}$ in Model $\mathrm{S}$.

The numerical solutions of the differential equations were obtained by the program of the modified Fowler-Warten method which included the restriction of non-negative concentrations of reactants (Aso et al., 1977). The "factor" in this program was fixed at 100 from preliminary experiments.

All computations were performed by a FACOM 230-75 digital computer in the Computer Center of Kyushu University.

\section{RESULTS}

\section{Relation between Model A and Model S}

As described above (see Computation), the $k_{8 \infty}$ and $k_{9 \infty}$ values were obtained from $P_{\infty}$ calculated for each of arbitrarily given $X_{0}$ values. The results are shown in Table 1. The computed results for Model $\mathrm{S}$ with approximated values of $k_{s}=1.0$ and $k_{9}=0.5$ are shown by curve $\mathrm{S}$ in Fig. 2, together with the results obtained for Model A (curve A). The latter is the same as curve B in the preceding paper (Okuyama et al., 1977). For the $P_{\infty}$ value, curve $\mathrm{S}$ coincided approximately with curve A. On the other hand, the two curves for the $Y_{\infty}$ value were roughly in parallel with each other in the range of $X_{0} \approx 3.5$. As a whole, it is concluded that Models $\mathrm{A}$ and $\mathrm{S}$ behave similarly.

\section{Effects of $K$ on Model $T$}

For Model T, the relationships between $E_{a \omega}$ and the input concentration were studied with changing $K$ values with fixing the $k_{\sharp}$ value at 1.0 . The results are shown in Fig. 3-a. When $K$ was 0.1 or above, the calculated $E_{\text {as }}$ value soon attained a saturated state, whereas under the conditions with $K \leq$ $0.01, E_{a \infty}$ depended upon the input $X_{0}$ linearly. Consequently, in the latter

Table 1. Calculated $k_{8 \infty}$ and $k_{9 \infty}$ values for Model A.

\begin{tabular}{rccc}
\hline$X_{0}$ & $P_{\infty}$ & $k_{8 \infty}=p-k_{5} P_{\infty}$ & $k_{9 \infty}=k_{6} P_{\infty} k_{7}$ \\
\hline 0.5 & 0.24 & 0.98 & 0.24 \\
1.0 & 0.46 & 0.95 & 0.46 \\
2.0 & 0.50 & 0.95 & 0.50 \\
3.5 & 0.50 & 0.95 & 0.50 \\
6.0 & 0.50 & 0.95 & 0.50 \\
10.0 & 0.50 & 0.95 & 0.50 \\
\hline
\end{tabular}




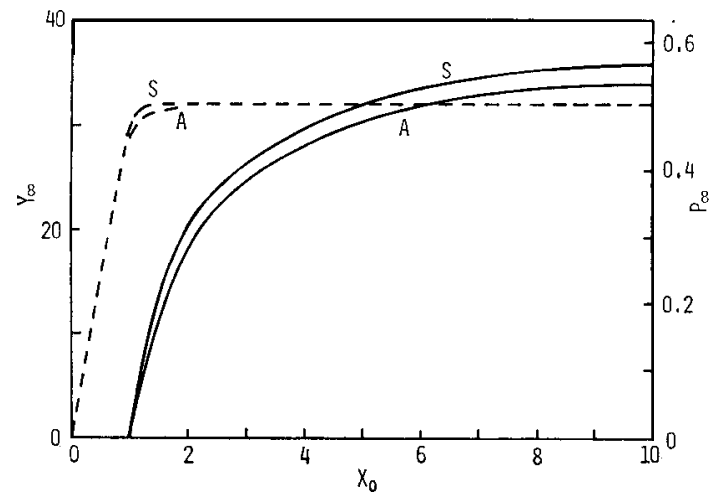

Fig. 2. Comparison of calculated results for Model $\mathrm{S}$ (curve $\mathrm{S}$ ) with those of curve A. Solid line is the $Y_{\infty}$ value and broken line is the $P_{\infty}$ value. Curve $A, p=1.0, k_{5}=0.1, k_{6}=1.0$ and $k_{7}=1.0$; curve $\mathrm{S}, k_{8}=1.0$ and $k_{9}=0.5$. The other parameters were $: q=0.05, k_{1}=400.0, k_{2}=35.0, k_{3}=5.0$ and $k_{4}=$ 2.0.

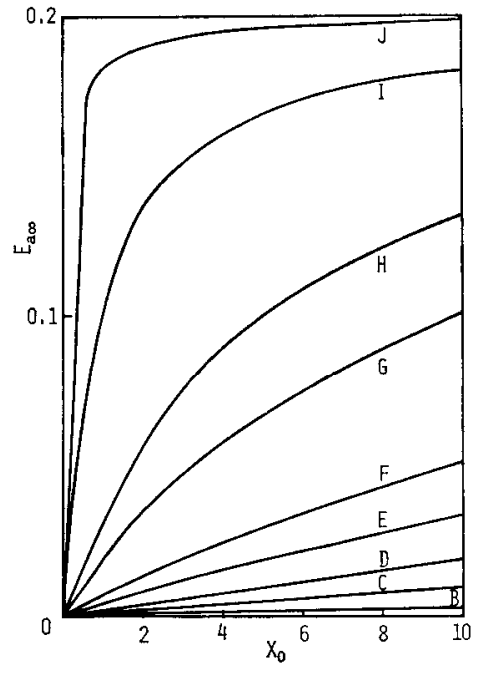

(a)

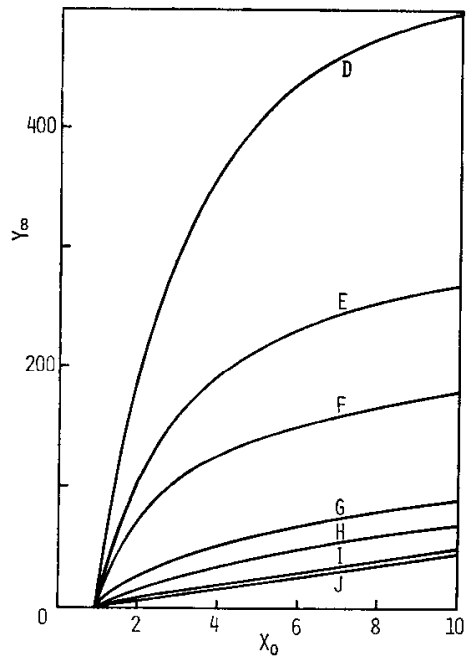

(b)

Fig. 3. Computed results for Model $\mathrm{T}$ with the changing $\mathrm{K}$ value. Curve $\mathrm{B}, \mathrm{K}=10^{-3}$; curve $\mathrm{C}, \mathrm{K}=\mathrm{O} .5 \times 10^{-2}$; curve $\mathrm{D}, K=10^{-2}$; curve $\mathrm{E}, \mathrm{K}=\mathrm{O} .2 \times 10^{-1}$; curve F, $K=1 / 3 \times 10^{-1}$; curve G. $K=10^{-1}$; curve H, $K=O .2$; curve I, $K=1.0$; curve J. $K=10.0$. The other parameters were $: k_{1}=400.0, k_{2}=35.0, k_{3}=$ $5.0, k_{4}=2.0, k_{8}=1.0$ and $k_{10}=1.0$.

(a) A relationship between $E_{a \infty}$ and $X_{0}$.

(b) A relationship between $Y_{\infty}$ and $X_{0}$.

case the ratio $X_{o} / E_{a \infty}$ had a value regardless of the input $X_{0}$ but unique for $\boldsymbol{K}$. 


\section{Relation between $K$ and $Y_{\infty}$}

The relationships between $Y_{\infty}$ and $K$ are shown in Fig. 3-b. When K was at the level of $10^{-2}$, the initial slope (at $X_{0}=1$ ) was sharp and the curve gradually approached to a saturated state. As the $K$ value increased, the initial slope of the curve became more gentle, causing a situation that the saturated state was not realized in the examined region of $X_{0}$.

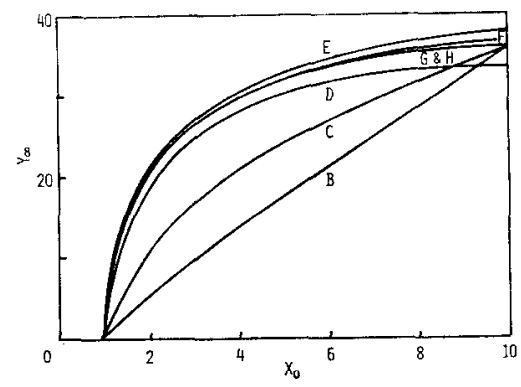

Fig. 4. Computed results for Model $\mathrm{T}$ with various sets of $K$ and $k$, , Curve B, $K=1$ and $k_{10}=1.37$; curve C, $K=10^{-1}$ and $k_{10}=2.5$; curve D, $K=10^{-2}$ and $k_{10}=14$; curve E, $K=0.5 \times 10^{-2}$ and $k_{10}=25$; curve F, $K=10^{-3}$ and $k_{10}=$ 125; curve G, $K=10^{-4}$ and $k_{10}=1250$; curve $\mathrm{H}, K=10^{-5}$ and $k_{10}=12500$. The other parameters were the same as those in Fig. 3.

\section{Relation between $K$ and $\boldsymbol{k}_{10}$}

By fixing the $k_{9}$ value at 0.5 and by substituting the $E_{a_{\infty}}$ values, which were calculated under the conditions with $X_{0}=10$ and varying $K$ values, into equation (5), the values of $k_{10}$ were obtained. Then simulation was performed for Model $\mathrm{T}$ by using each set of $K$ and $k_{10}$. The results are shown in Fig. 4. Approximately saturated curves were obtained when $\mathrm{K} \leqq 10^{-2}$ (see curves $\mathrm{D}$ to $\mathrm{H}$ ). The curves in the range of $K \leqq 10^{-4}$ in Model $\mathrm{T}$ (curves $\mathrm{G}$ and $\mathrm{H}$

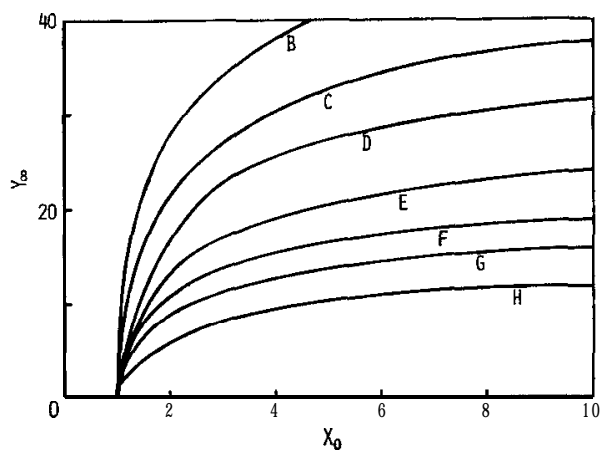

Fig. 5. Computed results for Model $\mathrm{T}$ with the changing $k_{10}$ value and a fixed value of $K=0.5 \times 10^{-2}$. Curve $\mathrm{B}, k_{10}=20$; curve C. $k_{10}=25$; curve D, $k_{10}=30$; curve E. $k_{10}=40$; curve F, $k_{10}=50$; curve G, $k_{10}=60$; curve $\mathrm{H}, k_{10}=$ 80. The other parameters were the same as those in Fig. 3. 
in Fig. 4) agreed precisely with the calculated curve for Model S (curve $\mathrm{S}$ in Fig. 2).

With a $K$ value below $10^{-6}$, the $k_{10}$ value obtained from equation (5) exceeded $10^{5}$; these values seem to be abnormally high for an enzymatic system.

\section{Effects of $\boldsymbol{k}_{10}$}

The effects of $k_{10}$ values on the threshold curve under a fixed value of $K$ $=O .005\left(k_{11}=0.1\right.$ and $\left.k_{12}=20.0\right)$ are shown in Fig. 5. All the curves obtained here had the same value of 1.0 for the threshold, and were substantially saturated. The relationship $k_{10} \propto 1 / Y_{\infty}$ holds when $X_{0}$ was 3.5 or above.

\section{DISCUSSION}

As is evident from Table 1 and Fig. 2, the $P_{\infty}$ value was saturated with $X_{0}$ in the region of $X_{n} \geqq 2.0$ and hence the relation $k_{9} \propto k$, was realized invariably with a proportional constant of $k_{6} P_{\infty}=0.50$. If $k_{8}$ is fixed at 0.95 , equations (1) and (2) would agree precisely at $X_{0} \geqq 2$. 0 . The values of $k_{8 \infty}$ and $k_{9 \infty}$ were in the range of $0.95 \leqq k_{3 \infty} \leqq 0.98$ and $0.24 \leqq k_{9 \infty} \leqq 0.50$ (Table 1) ; these values were approximated as $k_{3}=1.0$ and $k_{9}=0.5$ in order to be used for simulation of Model S. This approximation may cause a slight difference between curves A and S (Fig. 2). Nevertheless, the fundamental behavior of Model S was consistent with that of Model A. Therefore, it seemed quite reasonable to simplify and rewrite Model A into Model $\mathrm{S}$ in order to investigate the mechanism responsible for the saturated threshold responses.

As to Model $\mathrm{T}$, the relation of $E_{a} \propto X_{3}$ was maintained under the conditions with $K \leqq 0$. 01 as shown in Fig. 3-a, and similar (or the same) results as those for Model $\mathrm{S}$ were obtained as illustrated in Fig. 4 (curve D-H). Accordingly, provided that $10^{-5} \leqq K \leqq 10^{-2}$, Model $\mathrm{T}$ can be considered to serve as a practical model for study of feed-forward mechanism included in Model $\mathrm{S}$. Moreover, also in the above region of $K$, with $X_{0} \geqq 2.0$ and with other parameter values used in the present study, the following relationship is established from equations (1), (2) and (4):

$$
k_{10} \propto k_{9} \propto k_{7}
$$

and this relation would be confirmed by combining Fig. 10 of the previous paper (Okuyama et al., 1977) with Fig. 5 of the present paper.

From the above results, Model $\mathrm{T}$ is considered to be one of the model systems that can generate threshold responses and saturation in purely chemical reactions. Model $\mathrm{T}$ comprises a feed-forward composed of an equilibrating enzyme system and it is reasonable to expect that in order to get more effective feed-forward producing the saturation, a larger value is desirable for the equilibrium constant $K$. Nevertheless, contrary to this expectation, our results show that $K$ must be as small as possible for realizing the saturated threshold response. 


\section{REFERENCES}

Aso, Y., Y. Tsuji and K. Hayashi 1977 Application of the Fowler-Warten method to a stiff enzymatic reaction. J. Fac.Agr., Kyushu Univ., 21: 33-45

Lewis, J., J. M. W. Slack and L. Wolpert 1977 Thresholds in development. J. Theor. Biol., $65: 579-590$

Ličko, V. 1972 Some biochemical threshold mechanisms. Bull. Math. Biophysics, 34: 103112

Nimmo, I. A. and A. Bauermeister 1976 Co-operativity in the cyclic model for drug-receptor interaction. Biochem. Pharmacol., 25 : 1903-1905

Monod, J., J. Wyman and J. Changeux 1965 On the nature of allosteric transitions : a plausible model. J. Mol. Biol., 12: 88-118

Okuyama. K.. M. Okamoto. Y. Aso and K. Hayashi 1976 Studies on the threshold mechanism of enzymatic reaction. J. Fac. Agr., Kyushu Univ., 20: 87-95

Okuyama, K., M. Okamoto and K. Hayashi 1977 On the saturation of the threshold-type response in enzymatic reaction system. J. Fac.Agr., Kyushu Univ., 21: 67-77

Walter, C., R. Parker and M. Ycis 1967 A model for binary logic in biochemical systems. J. Theor. Biol., 15: 208-217 